\title{
Combining Models is More Likely to Give Better Predictions than Single Models
}

\author{
Xiaoping Hu, Laurence V. Madden, Simon Edwards, and Xiangming Xu
}

First author: State Key Laboratory of Crop Stress Biology for Arid Areas, College of Plant Protection, Northwest A\&F University, Yangling 712100, P. R. China; second author: Department of Plant Pathology, Ohio State University, Wooster, OH 44691; third author: Harper Adams University, TF10 8NB, Newport, Shropshire, UK; and fourth author: East Malling Research, East Malling, Kent, ME19 6BJ, UK. Accepted for publication 2 April 2015.

\begin{abstract}
Hu, X., Madden, L. V., Edwards, S., and Xu, X. 2015. Combining models is more likely to give better predictions than single models. Phytopathology 105:1174-1182.

In agricultural research, it is often difficult to construct a single "best" predictive model based on data collected under field conditions. We studied the relative prediction performance of combining empirical linear models over the single best model in relation to number of models to be combined, number of variates in the models, magnitude of residual errors, and weighting schemes. Two scenarios were simulated: the modeler did or did not know the relative of performance of the models to be combined. For the former case, model averaging is achieved either through weights based on the Akaike Information Criterion (AIC) statistic or with arithmetic averaging; for the latter case, only the arithmetic averaging is possible (because the relative model predictive performance is not known for a common dataset). In addition to two experimental

datasets on oat mycotoxins in relation to environmental variables, two datasets were generated assuming a consistent correlation structure among explanatory variates with two magnitudes of residual errors. For the majority of cases, model averaging resulted in improved prediction performance over the single-model predictions, especially when a modeler does not have the information of relative model performance. The fewer variates in the models to be combined, the greater is improvement of model averaging over the single-model predictions. Combining models led to very little improvement over individual models when there were many variates in individual models. Overall, simple arithmetic averaging resulted in slightly better performance than the AIC-based weighted averaging. The advantage in model averaging is also noticeable for larger residual errors. This study suggests that model averaging generally performs better than single-model predictions, especially when a modeler does not have information on the relative performance of the candidate models.
\end{abstract}

Much of applied research in agriculture is concerned with selecting models for describing observed data, testing hypotheses, and predicting future scenarios. Many criteria have been proposed to assist in model selection from both frequentist and Bayesian perspectives (Johnson and Omland 2004; Kadane and Lazar 2004); for example, Akaike information criterion (AIC), Schwartz criterion (also known as Bayesian information criterion), Mallows criterion, and the coefficient of determination $\left(R^{2}\right)$. When a hypothesis and its alternatives can be expressed explicitly as models, relative goodness-of-fit of these competing models to observed data can then be used as evidence for or against the hypothesis. For example, hypothesis testing, formulated in terms of model selection, has been used to analyze data on mark-recapture (Sillett and Holmes 2002) and molecular systematics (Felsenstein 2003). When observed data fail overwhelmingly to support any one of the competing models, model averaging may be used to estimate model parameters and make predictions based on various model-averaging schemes (Burnham and Anderson 2002; Hansen 2007; Hoeting et al. 1999).

It is sometimes not possible to formulate hypotheses about a complex problem into models because of our limited understanding. Furthermore, in applied research, hypothesis testing may not be the purpose; rather, researchers may like to develop an empirical model relating a response variable of interest to other variables without prior knowledge of the exact form of the model or explanatory variates to be included in the model. Such empirical models could then be used in the future as a basis of hypothesis

Corresponding author: X. Xu; E-mail address: Xiangming.xu@emr.ac.uk

http://dx.doi.org/10.1094/PHYTO-11-14-0315-R

(C) 2015 The American Phytopathological Society testing or prediction after further validation. One such example is the development and validation of models for predicting disease epidemics on crops based on environmental and other variables. These models are usually developed from data obtained from controlled-environment and field studies (De Wolf and Isard 2007; Hardwick 2006; Madden et al. 2007). Particularly for data collected under field conditions, how to construct a single "best" model with appropriate number of predictors is problematic. With a potentially very large number of correlated explanatory variates, there are numerous unknown alternative models that could be developed as a best model, all with similar levels of prediction accuracy. For instance, it is not uncommon to have more than 15 potential explanatory weather-related variates. Because of high collinearity among variates, the selection of variates in multiple regression analysis becomes both an important and problematic issue. Researchers often use automated stepwise multiple regression to select a best model, in spite of wide recognition of the major limitations of stepwise multiple regression (Hurvich and Tsai 1990; Johnson et al. 2004; Stephens et al. 2005; Steyerberg et al. 1999; Whittingham et al. 2006; Wintle et al. 2003). The chosen best model is often not explicitly compared with other alternatives; indeed, researchers usually would not see other alternative models with an automated approach. The main drawbacks of stepwise multiple regression include bias in parameter estimation, inconsistencies among model selection algorithms, an inherent problem of multiple hypothesis testing, invalid calculated $P$ values for the hypothesis tests, overfitting of the training data (at the expense of the testing data), and an inappropriate focus or reliance on a single best model when there are two or more models that have almost equal explanatory power. Some of the problems can be ameliorated by selecting models using test data (not used in estimating parameters) 
and by incorporating randomization steps in the model selection process (Hastie et al. 2009). However, uncertainty in model selection is still ignored when a single model is chosen and used (Yang 2003; Yuan and Yang 2005).

Recently, all-subsets logistic regression, with models selected based on test datasets not used in the parameter estimation, was used to predict the risk of Fusarium head blight (FHB) in cereals (Shah et al. 2013; Xu et al. 2013, 2014). These researchers showed that, for a given dataset, many models could explain the observed data with similar predictive power and that the best model varied greatly with subset samples in the dataset. Hence, there was inconsistency in the best model across subset samples, which is analogous to different models being appropriate for different studies. In addition to differences in hosts and pathogen virulence, this inconsistency in selecting the best model may partially explain why there are multiple predictive models published for a single pathosystem; for example, FHB in wheat (De Wolf et al. 2003; Landschoot et al. 2012; Moschini and Fortugno 1996; Moschini et al. 2001), apple scab (MacHardy 1996), and late blight of potato (Hardwick 2006).

Given the availability of multiple models for prediction, the question is whether and, if so, how to select a best one (or, indeed, to develop another one that fits new data better than published ones), or whether to combine models for prediction. Consider the following examples. Seventeen models were compared for their prediction of breast cancer risks (Meads et al. 2012); none of these models was able consistently to predict the risk accurately. Watershed models that predict flow, nitrogen, and phosphorus discharges were recently compared for their predictive powers (Boomer et al. 2013). No model consistently matched observed values better than the others; models that agreed best with the observations in one point often were among the worst models for another. Averaging model predictions improved overall accuracy in predictions, although averaging prediction was not the closest to the observed for every sample. Recently, Shah et al. (2014), in reanalyzing the FHB data with boosted regression trees, showed that model averaging could improve certain aspects of predictive accuracy compared with any single one of the boosted-regression-tree models that were averaged. Statisticians have advocated the use of model averaging (or ensemble modeling) to improve predictions (Exbrayat et al. 2013; Montgomery 2012).

As stated by Yuan and Yang (2005), "it is still unclear to a large extent when model combining [averaging] should be preferred" relative to best-model selection. These authors showed that, as expected, model averaging is preferred if there is uncertainty in the model selection process, as represented by the residual variance of a selected model. In botanical epidemiology, there is usually considerable uncertainty in the selected empirical models used for prediction (Shah et al. 2014; Xu et al. 2014); therefore, it is desirable to understand factors that jointly affect the improvement of prediction by averaging two or more models. In this article, we deal with scenarios typically found when attempting to predict plant disease epidemics with empirical linear models based on weather conditions. In addition to the level of the residual variance, we consider factors or combinations of factors that have not been considered previously in this context. Specifically, two situations were studied: in case 1, a modeler has access to all datasets (i.e., the modeler knows or has an estimate of the relative performance of alternative models in explaining the same set of observed data) and, in case 2 , a modeler can only access published best models without knowing their relative performance on common datasets. In case 2, this means that we only have several best models that were fitted and published based on different datasets rather than having access to the entire set of models and datasets as in case 1 . Thus, for case 1, the analyst has access to the measures that quantify goodness-of-fit of all candidate models to the data, and methods based on weighted averaging are then possible.

\section{THEORY AND APPROACHES}

Model averaging. When no single model is overwhelmingly supported by the data, it becomes problematic to choose one model over another. Model averaging provides a way to address this problem. Parameter estimates or predictions obtained by model averaging are often robust in the sense that they reduce model selection bias and account for model selection uncertainty (Burnham and Anderson 2002; Hansen 2007; Yang 2003; Yuan and Yang 2005).

Assuming there are $K$ candidate models and $N$ explanatory variates (predictors or predictor variables), the $j$ th model can be written as

$$
y_{i}=\mu_{j i}+\varepsilon_{j i}=\sum_{l=1}^{N} \theta_{j l} x_{l}+\varepsilon_{j i}
$$

where $y_{i}$ is the $i$ th observed value of the response, $\mu_{j i}$ is the predicted average response for the $i$ th observation by the $j$ th model, $\theta_{j l}$ is the parameter estimate for the explanatory variate $x_{l}(l=1, \ldots, N)$ for the $j$ th model, and $\varepsilon_{j i}$ is the residual error term for the $i$ th observation of the $j$ th model. When averaging all $K$ models to obtain the average prediction, one can write:

$$
\mu_{i}=\sum_{j=1}^{K}\left(w_{j} \sum_{l=1}^{N} \theta_{j l} x_{l}\right)
$$

where $w_{j}$ is the weight for the $j$ th model

$$
\left(\sum_{j=1}^{K}\left(w_{j}\right)=1.0\right)
$$

Thus, average parameter estimate for the $l$ th variate is

$$
\bar{\theta}_{l}=\sum_{j=1}^{K}\left(w_{j} \theta_{j l}\right)
$$

The model weight $\left(w_{j}\right)$ can be calculated by various means, such as Bayesian model weights derived from posterior model probabilities (Raftery et al. 1997), exponential AIC weights (Akaike 1980; Burnham and Anderson 2002), or through more complex functions (Yuan and Yang 2005). A simple model averaging scheme is to use equal weight for all models (i.e., $w_{j}=1 / K$ ).

The different models may have one or more different $x$ variates. One can base the parameter averaging just on the variables in the available models (e.g., if $x_{3}$ appears in models 1 and 2 and not the rest, then the average for $\theta_{3}$ is over these two models). However, if a variate ( $\operatorname{such}$ as $x_{3}$ ) is not in a linear model, then this is equivalent to the corresponding parameter being equal to 0 (e.g., $\theta_{j 3}=0$ ). Thus, it is more common to base the averaging of each parameter on all the models, using 0 for the parameter $\left(\theta_{j l}\right)$ in a given model if the corresponding variate is not present. The latter approach for linear models is equivalent to simply averaging the predictions $\left(\mu_{j i}\right)$ from all of the individual models (without explicit consideration of the parameters); this approach was used in the present study. Thus, model averaging can be seen as doing at least two different things. It attenuates the extremes in parameter estimates in individual models, usually by shrinking the average estimate toward 0 (by the averaging of zero estimates [for models without particular variates] with the nonzero estimates), and it incorporates information from more variates in the predictions. The latter is done by the incorporation of more variates in the final combined model (albeit with parameter estimates smaller than in a single model).

To use differential weights for different models, it is essential to have some measures quantifying the relative performance of different models. Thus, in the present study, weighted model averaging was possible only for case 1 , whereas equal weight averaging was applied to both case 1 and 2 . We used the exponential AIC weight in the present study (Burnham and Anderson 2002). Assuming that the AIC values of the top $K$ models were denoted as $\mathrm{AIC}_{1}, \mathrm{AIC}_{2} \ldots \mathrm{AIC}_{\mathrm{K}}$ and that the first model was the best model, the quantity 


$$
R L_{j}=\mathrm{e}^{\frac{1}{2}\left(\mathrm{AIC}_{1}-\mathrm{AIC}_{j}\right)}
$$

is the likelihood of the $j$ th model relative to the best one. Then, $w_{j}$ is calculated as

$$
w_{j}=\frac{R L_{j}}{\sum_{1}^{K} R L_{j}}
$$

Datasets. Four datasets were used to study prediction performance of using single models and combining them. Two datasets were from a published study of mycotoxins in oat (Xu et al. 2014) and the other two datasets were artificially generated with known correlation structures among variates.

Oat mycotoxin data. Details about the oat datasets were reported previously (Xu et al. 2014). The response variate was the total concentration of HT2 and T2 mycotoxins quantified on oat grain (on the scale of natural logarithm), with the effects of cultivars and number of cereal crops in previous seasons removed. Seventeen weather variables were summarized over a window length of 20 days for each of the three periods: late May, mid-June, and late August. These three periods were shown by window-pane analyses to be the most important periods when weather conditions are most associated with mycotoxin accumulation. In total, there were 201 samples. The first dataset (Oat_A) used in this study is the complete set summarized for the window length of 20 days (i.e., 201 samples, each with 51 weather variables in addition to the toxin concentration). The second dataset (Oat_P) consisted of all 201 samples with only the 17 explanatory variables for the late-May period (in addition to the toxin data).

Simulated datasets. Two datasets were simulated using the $\mathrm{R}$ software (version 3.03, 64 bits) in two steps. A set of 12 explanatory variates was generated, with a consistent correlation structure among the variates across samples. Then, a response variate was derived from 6 of the 12 explanatory variates, with an additive error added: this error was drawn from one of two normal distributions differing in their variance.

Correlated and normally distributed explanatory variables $(D)$ were generated using the Cholesky decomposition (Ralston and Rabinowitz 1978). If $C$ is the correlation matrix, then $U^{T} U=C$, where $U$ is an upper triangular matrix. If $X$ is a matrix of independent random variables with a standard normal distribution, then $D=X U$ is a matrix of normally distributed variables with correlation defined by $C$. Below, we use subscripts to matrices to designate different specific simulation situations, and numbers in square brackets to indicate a different column of the matrices.

Two six-variable correlation matrices were used:

$$
\begin{aligned}
C_{1} & =\left(\begin{array}{cccccc}
1 & -0.4 & 0.35 & 0.1 & 0.05 & 0.05 \\
-0.4 & 1 & 0.25 & 0.15 & 0.1 & 0.05 \\
0.35 & 0.25 & 1 & 0.2 & 0.15 & 0.1 \\
0.1 & 0.15 & 0.2 & 1 & 0.1 & 0.05 \\
0.05 & 0.1 & 0.15 & 0.1 & 1 & 0 \\
0.05 & 0.05 & 0.1 & 0.05 & 0 & 1
\end{array}\right) \text { and } \\
C_{2} & =\left(\begin{array}{ccccccc}
1 & 0.8 & 0.7 & 0.2 & 0.1 & 0.1 \\
0.8 & 1 & 0.5 & 0.3 & 0.2 & 0.1 \\
0.7 & 0.5 & 1 & 0.4 & 0.3 & 0.2 \\
0.2 & 0.3 & 0.4 & 1 & 0.2 & 0.1 \\
0.1 & 0.2 & 0.3 & 0.2 & 1 & 0 \\
0.1 & 0.1 & 0.2 & 0.1 & 0 & 1
\end{array}\right)
\end{aligned}
$$

The magnitude of these correlations was based on the correlation commonly observed among weather-related variates often used in epidemiological studies. Let $X 1$ and $X 2$ represent matrices of 200 rows and six columns (i.e., six variates), with each element randomly drawn from a standard normal distribution, $N(0,1)$. Then, $D 1$ and $D 2$ are matrices determined from $X 1$ and $X 2$ and the Cholesky decomposition of the matrices $C 1$ and $C 2$, respectively; thus, $D 1$ and $D 2$ each contained 200 rows and six columns. Finally, a response variate $(y)$ was calculated as

$$
y=D 1[, 1]-D 1[, 2]+D 1[, 3] / 2+D 2[, 1]-D 2[, 2]+D 2[, 3] / 2+\varepsilon
$$

where the $[, l]$ notation is used to represent all the observations of the variates of the $l$ th column of the target matrix, and $\varepsilon$ is a normally distributed error randomly drawn from either $N(0,3)$ (dataset $1-\mathrm{DS} 1)$ or $N(0,1)$ (DS2). Although there were two groups of explanatory variates in the true model (variables 1 to 3 and 4 to 6 ), with correlations within the groups (corresponding to $C 1$ and $C 2$, respectively) and zero correlation between groups, the correlation among the 12 variates is consistent across the 200 samples. Therefore, the DS1 (DS2) consisted of $y$ and 12 column vectors in $D 1$ and $D 2$ as explanatory variates. Although only 6 of the 12 variates were actually used to generate $y$ (equation 2), the other variables affect results because of the intervariate correlations.

Resampling and combining model predictions. For each of the four datasets (Oat_A, Oat_P, DS1, and DS2), two separate studies were conducted to simulate whether a modeler has access to all the data.

Case 1: A modeler has access to all datasets. The following steps were used to generate single or combined model predictions:

(1) The entire dataset was randomly divided into two equal parts (S1 and S2); for Oat_A and Oat_P, S1 and S2 had 101 and 100 samples, respectively;

(2) For a given number of explanatory variates (from one to six) in the estimated linear regression models, the R "subselect" package was applied to S1 (the training dataset) to obtain the best five models based on the coefficient of determination $\left(R^{2}\right)$. Any two of these five models will not contain the same set of explanatory variates.

(3) To predict the response variate in S2 (the test dataset), the top two, three, four, or five models for a given number of explanatory variates (one to six) were combined either as an arithmetic average or based on exponential AIC weight.

(4) Finally, the agreement between observed and predicted values for $\mathrm{S} 2$ by the best model and by combining the top two, three, four, or five models via arithmetic or weighted averaging was assessed in terms of (i) average absolute prediction error $[=$ mean $(\mid$ predicted-observed $\mid)]$, (ii) average prediction error [= mean(predicted-observed)], (iii) variance of prediction errors [= variance(predicted-observed)], and (iv) correlation between predicted and observed values.

(5) Processes 1 through 4 were repeated 200 times; that is, a new dataset (S1, hence $\mathrm{S} 2$ ) was generated from a given dataset (Oat_P, Oat_A, DS1, and DS2) 200 times by randomly assigning all the observations in the dataset to $\mathrm{S} 1$ and S2 (sampling without replacement; that is, permutation) and the model selection and prediction assessment were carried out for each permutated S1 and S2 dataset.

Case 2: A modeler cannot access all the datasets. The following steps were used to simulate this scenario:

(1) A complete dataset was randomly divided into three equal parts (S1, S2, and S3); for DS1 and DS2, S1 and S2 each had 67 samples and S3 had 66 samples;

(2) The subselect package was used to derive two best models for a given number of explanatory variates (one to six), one from $\mathrm{S} 1$ and the other from $\mathrm{S} 2$ (hence their relative performance on common data are unknown, given that they were fitted to different datasets);

(3) These two best models were used to predict the response variate in S3 using arithmetic averaging. These models may contain the same set of explanatory variates but with different parameter estimates; 
(4) The agreement between observed and predicted (based on the two individual best models or their arithmetic average) was assessed as for case 1 .

(5) Steps 1 through 4 were repeated 200 times as for case 1.

Similarly, further simulation was done to combine three best models derived from three different subdatasets. In this scenario, a complete dataset was randomly divided into four (S1, S2, S3, and S4) subsets: the first three were used to derive the three best models (one from each subset) for a given number of variates $(N V)$ and $\mathrm{S} 4$ was used to evaluate their predictions. For Oat_A and Oat_P, S1, S2, and S3 each had 50 samples and S4 had 51 samples.

Data analysis. The effect of several simulation factors on the relative performance of model averaging and single-model prediction was quantified as the proportion of sum of squares accounted for by their main effects and interactions in analysis of variance (ANOVA). Although four measures of agreement between predicted and actual data were outputted for every random permutation, only detailed results of the correlation $(r)$ between the predicted and observed values were presented because preliminary analysis suggested that the main conclusions drawn from these four individual variables were nearly identical.

Two summary variables were analyzed in ANOVA across datasets. For each permutation, percentage of improvement $(P I)$ of model averaging over the single-model predictions was calculated as $P I=100\left(r_{a}-r_{s}\right) / r_{s}$ for case 1 and $P I=100\left[r_{a}-\max \right.$ $\left.\left(r_{s}\right)\right] / \max \left(r_{s}\right)$ for case 2 , where $r_{a}$ and $r_{s}$ were correlation coefficients for model-averaging and single-model prediction (where single is for the best), respectively, and $\max \left(r_{s}\right)$ was the maximum $r_{s}$ of the two or three best models for the test datasets in case 2. Average PI was calculated for 200 permutations for each dataset. The second summary variable was the percentage (NT) of 200 permutations when $r$ from model averaging was at least as good as the singlemodel predictions (i.e., $r_{a} \geq r_{s}$ for case 1 and $r_{a} \geq \max \left[r_{s}\right]$ for case 2 ). Four separate ANOVA were applied to $P I$ and NT corresponding to two groups of datasets (oat and simulated data), each analyzed for case 1 and 2. For case 1 of the oat data, there were four factors: (i) datasets (Oat_P versus Oat_A), (ii) $N V$ in the model, (iii) number of models $(N M)$ to be combined, and (iv) averaging scheme ( $A W$ : AIC weighted or simple arithmetic averaging); for case 2, there were three factors: (i) datasets, (ii) $N V$, and (iii) $N M$ (i.e., two or three). For the simulated data, factors in the ANOVA were the same as for the oat data, except that residual variance $(R V)$ is analogous to the dataset factor.

Because statistical significance was not our primary interest (rather, we were interested in the relative differences in prediction performance and percentage variances accounted for by factors studied), data (i.e., $r, P I$, and NT) were not transformed before ANOVA and hypothesis testing was not done.

$\mathrm{R}$ code for the components of the simulation, sampling, and analysis is available from the corresponding author on request.

\section{RESULTS}

Overall results. Average absolute prediction errors were positively correlated with the variance of prediction errors $(>0.88)$ and negatively with the correlation between predicted and observed values, but not significantly correlated with average prediction errors. Average prediction errors were close to zero over 200 simulated runs for all combinations of simulation factors, indicating overall absence of biases in predictions.

There was large variation in $r$ among 200 permutations for a given combination of simulation factors; an example is shown in Figure 1 (Oat_A for $N V=3$ ). There were many situations where $r$ was close to zero; indeed, $r$ was negative (as low as -0.2 ) for several permutations. Model averaging appeared to reduce the extent of this variability, particularly for the simulated data (individual data not shown).

For both case 1 and 2, model averaging performed better than single-model predictions (Table 1); overall improvement in $r(P I)$ was greater for case 2 (approximately 14.1\%) than for case 1 (approximately 3.3\%) (Fig. 1A versus B). There were only small differences in $r$ between arithmetic and weighted averaging, particularly for the two oat datasets (Table 1). On the other hand, weighted averaging led to greater $N T$ than arithmetic averaging, particularly for DS2 (Table 1): NT > 80\%. Average correlation for
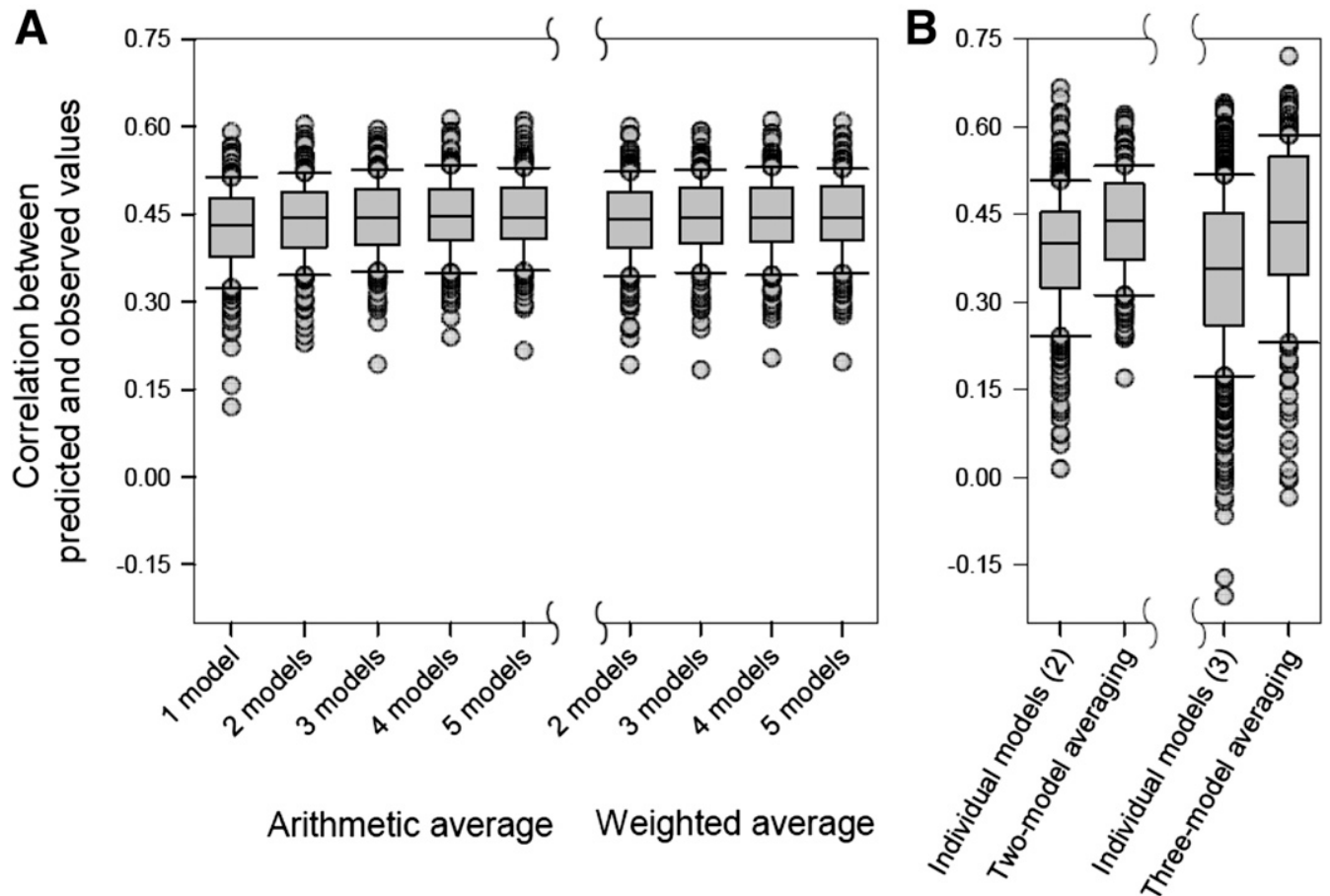

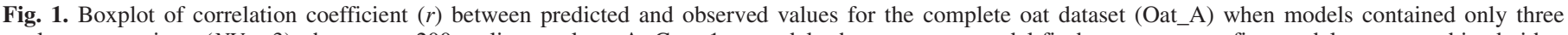

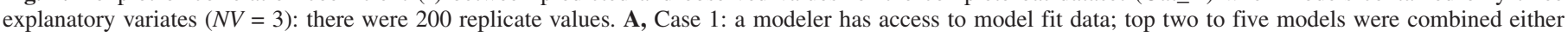

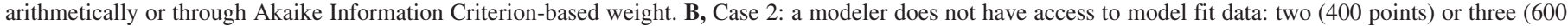
points) best models, one from a different group of observations, were combined arithmetically to provide predictions. 
DS2 was 0.817 and 0.791 for arithmetic and weighted averaging, respectively; this was high relative to the true underlying correlation for the generating model (0.892).

For case 2, PI was greater for three-model averaging (approximately 18.9\%) than for two-model averaging (approximately $9.9 \%$ ) (Table 1). For DS2 (i.e., for smaller residual variance), $N T$ was greater for three-model averaging than for two-model averaging; the opposite was true for the other three datasets (Table 1). In all cases, the performance (i.e., $r$ ) of model averaging was always greater than the worst-performing single model.

Prediction performance over all datasets. Oat data: Case 1. Approximately 27.2 and $10.6 \%$ of the total variability in the overall improvement in $r$ (i.e., $P I$ ) of model averaging over single-model prediction were due to the main effect of datasets (Oat_A versus Oat_P) and $N V$ (Table 2), respectively. Average PI was 5.0 and $1.2 \%$ for Oat_A and Oat_P, respectively. Increasing $N V$ from 1 to 2 increased $P I$ from 1.0 to $4.9 \%$ and further increase in $N V$ actually led to gradual decline in $P I$ (Fig. 2A). The overall effects due to the main effect of number of averaged models $(N M ; 1$ to 5$)$ and $A W$ (weighted versus arithmetic averaging) on $P I$ were small. The interaction between datasets (Oat_P versus Oat_A) and $N V$ accounted for $34.4 \%$ of the variability in PI (Table 2). Increasing $N V$ led to gradual decline in PI for Oat_A but, for Oat_P, increasing $N V$ from 1 to 2 led to a large increase in $P I$ and further increase in $N V$ resulted in greater reduction in PI than for Oat_A (Fig. 2B).

Most variability in $N T$ was due to the main effect of datasets $(33.0 \%)$ and $N V(23.4 \%)$, and their interactions (38.8\%) (Table 2). NT was greater for Oat_A (72.4\%) than for Oat_P (50.2\%). Overall, $N T$ initially increased sharply when $N V$ increased 1 to 2 and then decreased gradually (Fig. 2C). This initial increase in $N T$ with increasing $N V$ from 1 to 2 resulted entirely from the increase observed for Oat_P (Fig. 2D).

Oat data: Case 2. The main effect of NM (two- versus threemodel averaging), datasets (Oat_A versus Oat_P), and $N V$ accounted for $58.2,19.2$, and $14.1 \%$ of the total variation in PI (Table 3), respectively. $P I$ was 24.1 and $12.4 \%$ for three- and two-model predictions, respectively. PI was greater for Oat_A (21.6\%) than for Oat_P $(14.9 \%)$. Increasing $N V$ resulted in greater $P I$, especially from $N V=1(13.5 \%)$ to $3(18.2 \%)$. Interactions among factors were small.

$N T$ was largely affected by the main effect of $N V$ and its interaction with the datasets. The former explained nearly $78 \%$ of the total variability in NT and the latter close to $11 \%$ (Table 3 ). NT decreased sharply when $N V$ increased from 1 to 2 and then increased gradually with further increase in $N V$ (Fig. 3A). However, there was no simple trend in the interaction between $N V$ and the datasets (Fig. 3B).

Simulated data: Case 1. The main effect of $N V$ and $A W$ (weighted or arithmetic average) accounted for 43.2 and $9.6 \%$ of the total variability in $P I$ (Table 2), respectively. $P I$ decreased from $10.0 \%$ to nearly zero when $N V$ increased from 1 to 6 ( $P I$ was close to zero for $N V>3)$. $P I$ values were 5.2 and $1.9 \%$ for arithmetic averaging and weighted averaging, respectively. The interaction term between $A W$ and $N V$ accounted for $26.7 \%$ of the total variation in PI (Table 2). Large $P I$ was limited mainly to $N V=1(17.6 \%)$ or $2(8.8 \%)$ for arithmetic averaging; for all other combinations of $N V$ and $A W, P I$ was $<5 \%$ (Fig. 4A).

The main effect of $N V$ and $A W$ accounted for 66.9 and $7.1 \%$ of the total variability in $N T$ (Table 2), respectively. $N T$ decreased from 92.8 to $34.4 \%$ when $N V$ increased from 1 to 6 . Weighted averaging resulted in $N T=76.2 \%$ compared with $63.9 \%$ for arithmetic averaging. Interactions between $R V$ and $N V$ accounted for $16.8 \%$ of the total variability in PI (Table 2); NT was greater for smaller $R V$ than for the large $R V$ when $N V=1,2,4$, and 5, and the opposite was true for $N V=3$ and 6 (Fig. 4B).

Simulated data: Case 2. The main effect of $N V, R V, N M$ accounted for $10.4,64.4$, and $16.8 \%$ of the total variability in $P I$

TABLE 2. Percentage of the variance in the two summary variables, percentage of improvement $(P I)$ and percentage of 200 permutations when $r$ from model averaging was at least as good as the single-model predictions $(N T)$, on the relative performance of model averaging and single-model prediction summarized over 200 simulation runs accounted for by simulation factors and their interactions ${ }^{\mathrm{a}}$

\begin{tabular}{|c|c|c|}
\hline Data & $P I$ & $N T$ \\
\hline \multicolumn{3}{|l|}{ Oat data } \\
\hline Datasets & 27.16 & 33.01 \\
\hline Number of models (NM) & 2.07 & 1.45 \\
\hline Weighted versus averaging (AW) & 0.02 & 1.22 \\
\hline Number of variates $(\mathrm{NV})$ & 10.61 & 23.39 \\
\hline Datasets $\times \mathrm{NM}$ & 1.39 & 0.53 \\
\hline Datasets $\times$ AW & 3.16 & 0 \\
\hline $\mathrm{NM} \times \mathrm{AW}$ & 0.04 & 0.03 \\
\hline Datasets $\times \mathrm{NV}$ & 34.36 & 38.84 \\
\hline $\mathrm{NM} \times \mathrm{NV}$ & 0.88 & 0.67 \\
\hline $\mathrm{AW} \times \mathrm{NV}$ & 3.43 & 0.13 \\
\hline \multicolumn{3}{|l|}{ Simulated data } \\
\hline Residual variance (RV) & 2.97 & 0.23 \\
\hline NM & 2.53 & 0.32 \\
\hline AW & 9.63 & 7.09 \\
\hline NV & 43.21 & 66.85 \\
\hline $\mathrm{RV} \times \mathrm{NM}$ & 0.15 & 0.03 \\
\hline $\mathrm{RV} \times \mathrm{AW}$ & 0.16 & 1.8 \\
\hline $\mathrm{AW} \times \mathrm{NM}$ & 1.31 & 0.16 \\
\hline $\mathrm{RV} \times \mathrm{NV}$ & 1.28 & 16.82 \\
\hline $\mathrm{NV} \times \mathrm{NM}$ & 6.11 & 0.43 \\
\hline $\mathrm{AW} \times \mathrm{NV}$ & 26.72 & 0.84 \\
\hline
\end{tabular}

a This is the scenario where a modeler has access to all datasets and, hence, it was possible to use weighted averaging (i.e., case 1).

TABLE 1. Average correlation $(r)$ between the observed and predicted values using single-model and model averaging prediction, percentage of improvement $(P I)$ of model averaging over the single-model predictions in $r$, and percentage of simulations $(N T)$ where $r$ from model-averaging is greater or equal to the best of single models ${ }^{\mathrm{a}}$

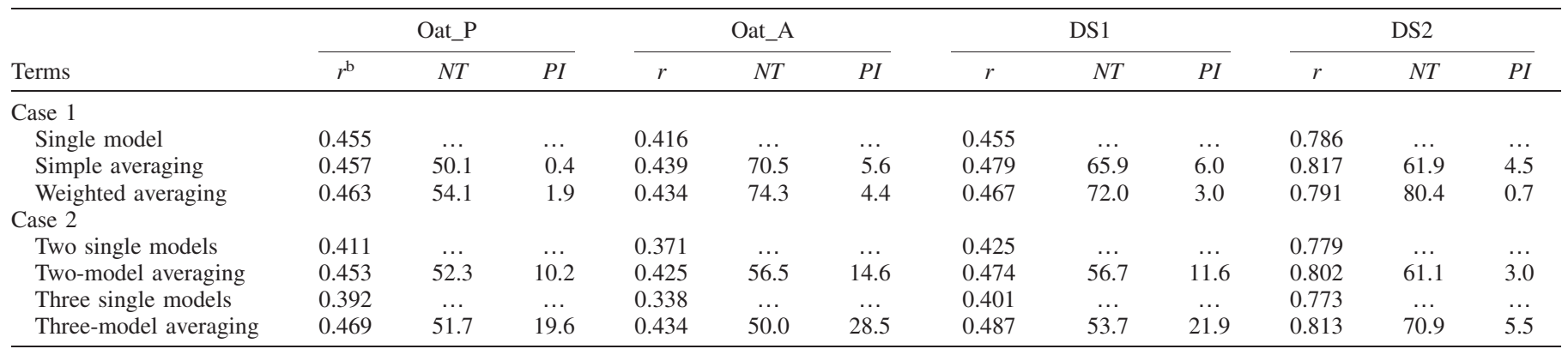

${ }^{\text {a }}$ For case 1, all models to be combined were fitted to the same dataset; for case 2, only the best models fitted to different datasets were combined. Partial oat data (Oat_P) only contained 17 of the 51 explanatory variables; the full oat data (Oat_A) contained all the 51 explanatory variables. DS1 and DS2 were two simulated datasets (equation 2).

${ }^{\mathrm{b}}$ Correlation between the right hand side of equation (2) and the response variable was 0.602 and 0.892 for DS1 and DS2, respectively. Note: the residual variance of equations 2 was 3 for DS1 and 1 for DS2. 
(Table 3), respectively. Increasing $N V$ from 1 to 6 decreased $P I$ from 12.2 to $7.6 \%$. Improvement of model averaging over single best models was greater for the larger $R V(P I=16.8 \%)$ than for the smaller $R V(P I=4.3 \%)$. Similarly, $P I$ was greater when combining three models than two models: $P I=13.7$ versus $7.3 \%$.

The main effect of $N V$ and $R V$ accounted for 63.8 and $11.1 \%$ of the total variability in $N T$, respectively (Table 3 ): increasing $N V$ or $R V$ led to lower $N T$. However, the exact $N T$ values depended on the interaction between $N V$ and $R V$ (accounting for $15.4 \%$ of the variation) (Table 3): $N T$ was greater for the smaller $R V$ when $N V<5$.

\section{DISCUSSION}

Deriving a single best empirical predictive model from an observed dataset can present a serious challenge because there may be several alternative models with similar explanatory powers (e.g., for predicting FHB disease) (Landschoot et al. 2012; Shah et al. 2013; Xu et al. 2013, 2014). To overcome this, model averaging is often proposed to estimate model parameters and make predictions based on various averaging schemes (Burnham and Anderson 2002; Exbrayat et al. 2013; Hansen 2007; Hastie et al. 2009; Hoeting et al. 1999; Montgomery 2012; Yang 2003). Several recent studies from disparate disciplines have shown that no single empirical model is consistently able to provide accurate prediction (Boomer et al. 2013; Meads et al. 2012). We demonstrated here that combining models leads to better predictions than individual models in the majority of situations typically faced by plant disease epidemiologists. In addition to the increased overall prediction accuracy, the variance in the prediction errors was always smaller for model averaging than for single-model prediction (results not shown),

TABLE 3. Percentage of the variance in the two summary variables, percentage of improvement $(P I)$ and percentage of 200 permutations when $r$ from model averaging was at least as good as the single-model predictions $(N T)$, on the relative performance of model averaging and single-model prediction summarized over 200 simulation runs accounted for by simulation factors and their interactions ${ }^{\mathrm{a}}$

\begin{tabular}{lcc}
\hline Data & $P I$ & $N T$ \\
\hline Oat data & & \\
Number of models $(\mathrm{NM})$ & 58.21 & 3.49 \\
Datasets & 19.19 & 0.29 \\
Number of variates $(\mathrm{NV})$ & 14.14 & 77.9 \\
$\mathrm{NM} \times$ Datasets & 2.15 & 1.88 \\
Datasets $\times \mathrm{NV}$ & 2.06 & 10.72 \\
$\mathrm{NM} \times \mathrm{NV}$ & 3.69 & 4.05 \\
Simulated data & & \\
$\mathrm{NM}$ & 16.83 & 1.18 \\
$\mathrm{Residual}$ variance $(\mathrm{RV})$ & 64.37 & 11.13 \\
$\mathrm{NV}$ & 10.36 & 63.8 \\
$\mathrm{NM} \times \mathrm{RV}$ & 6.26 & 3.86 \\
$\mathrm{NM} \times \mathrm{NV}$ & 0.59 & 2.55 \\
$\mathrm{NV} \times \mathrm{RV}$ & 1.39 & 15.41 \\
\hline
\end{tabular}

a This is the scenario where a modeler cannot access all the datasets and, hence, only arithmetic averaging was used (i.e., case 2).

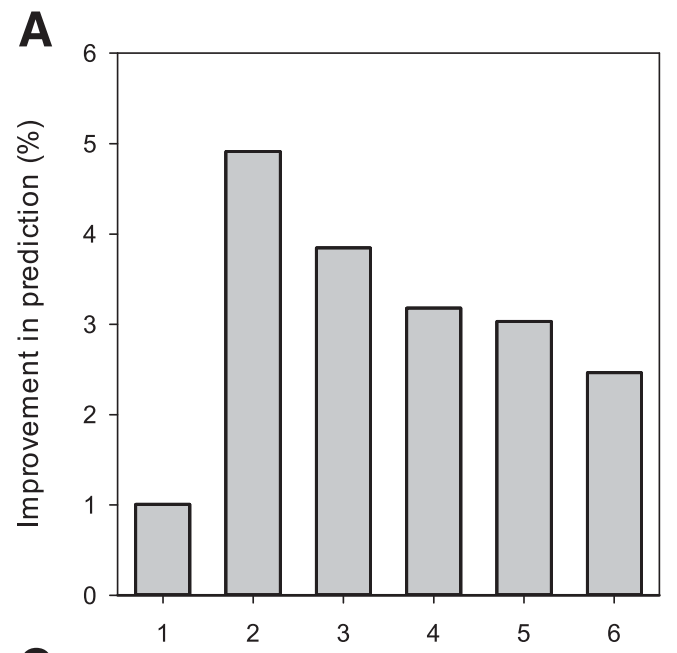

B
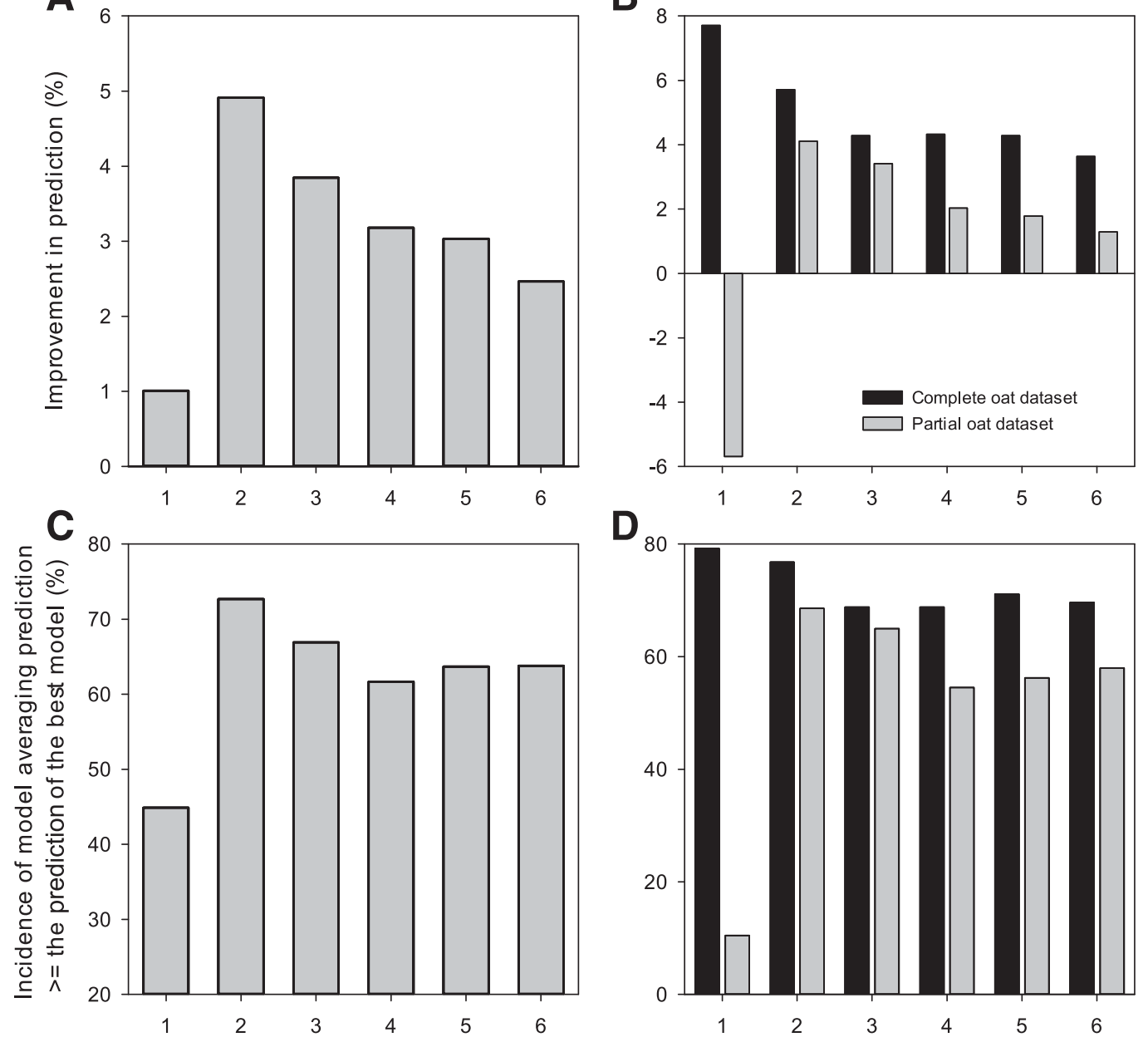

Number of variates in models

Number of variates in models

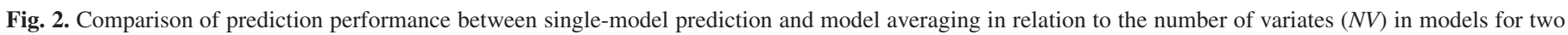

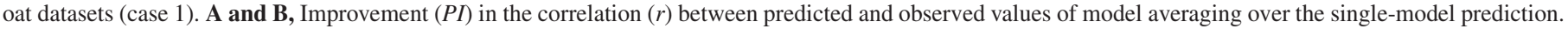
C and D, Overall incidence $(N T)$ when the correlation $(r)$ for model averaging was at least as good as the best model. 
especially when combining models derived from different datasets. Degree of improvement in prediction accuracy by combining models over individual models depended on several factors ( $N V$ in the model, $N M$ to be combined, and the size of residual errors), which can all be interpreted in terms of their influences on the reliability of individual models. We are not aware of other investigations where these factors were simultaneously explored in terms of the accuracy of a single selected model versus an average of multiple models, especially for situations where model performance on a common dataset is not known before the averaging. In some situations we explored, however, the improvement in accuracy was small.

The most important factor affecting the relative performance of model averaging relative to individual models was the $N V$ included in the models. $N V$ in the models is most influential when combining models derived from the same dataset (i.e., case 1). Under case 1, combining models is essentially similar to adding more variates in a model when $N V$ in a model is reasonably low, which is probably the main reason for increased prediction performance from model averaging. In terms of equation 1 , model averaging would be primarily adding nonzero $\theta_{l}$ parameters (for the added $x_{l}$ variates). For models with the same number of explanatory variates derived from the same dataset using the subselect package in R, they differ in at least one variate (by definition). Thus, for example, combining two one-variate models is equivalent to having a model with two variates, and combining two two-variate models is equivalent to having a model with at least three variates. That is, there are at least three unique explanatory variates in the averaged model but the possible variate in common will have a different parameter estimate depending on the other variates. If the additional variate is important, then model averaging will result in increased prediction power, with the extent of the improvement depending on the importance of the additional variate. With more variates already included in a model, any additional variate is expected to have reduced importance, agreeing with the obtained result that increasing $N V$ in individual models led to a reduced improvement of model averaging. For a given underlying data-generating
A

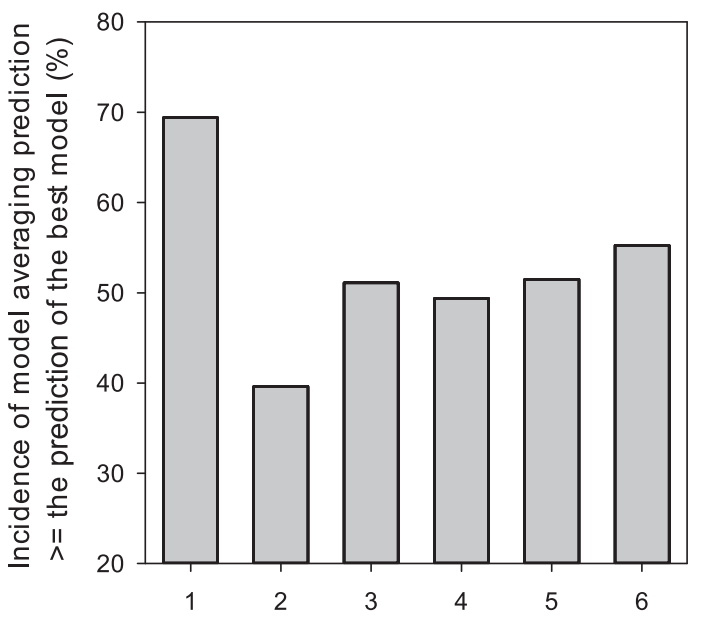

Number of variates in models
B

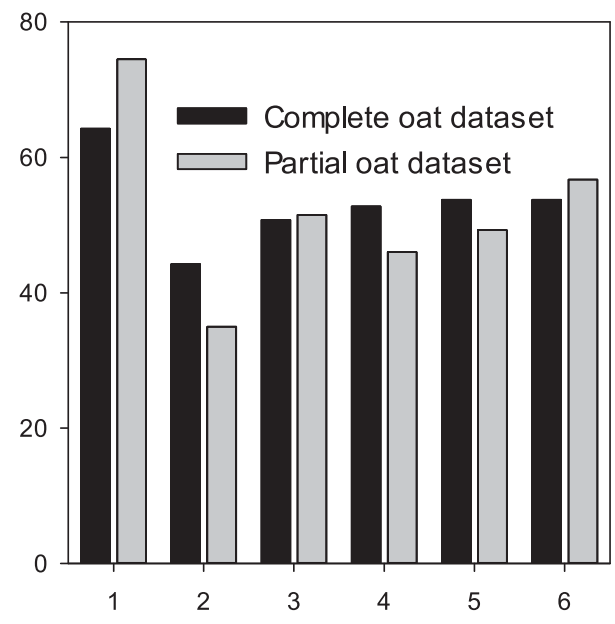

Number of variates in models

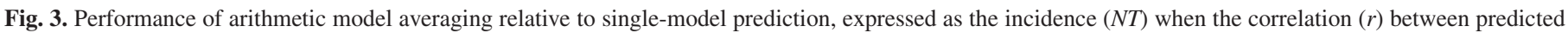
and observed values from model averaging was at least as good as the best from two or three best models for the oat datasets (case 2).

\section{A}

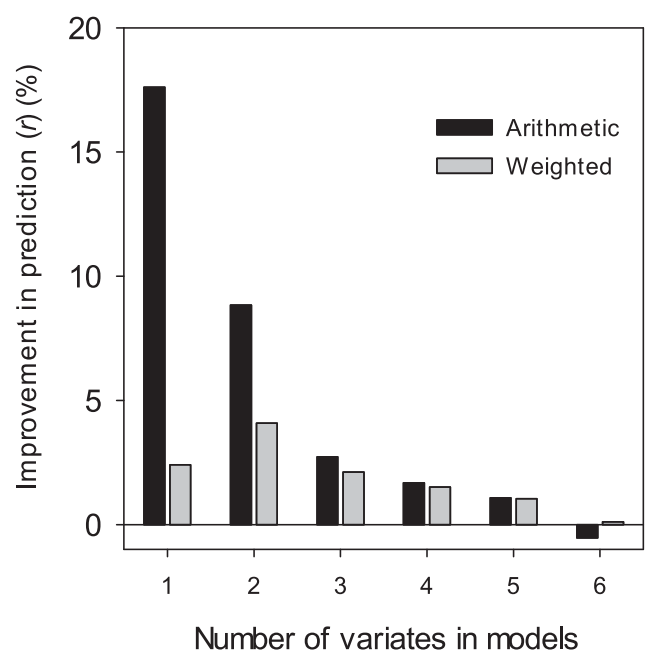

B

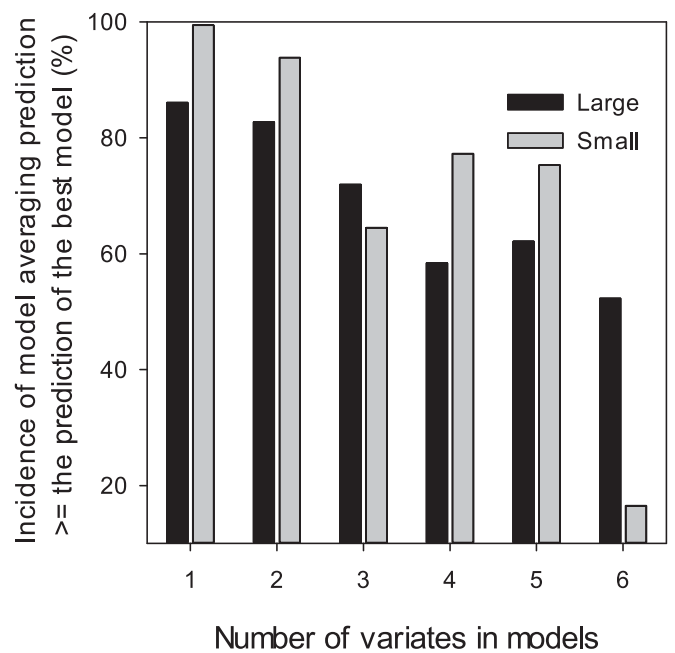

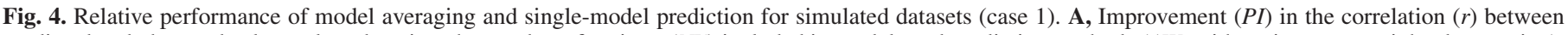

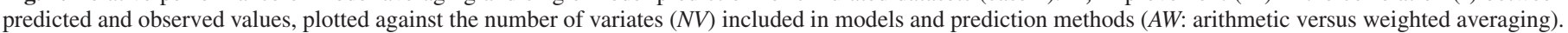

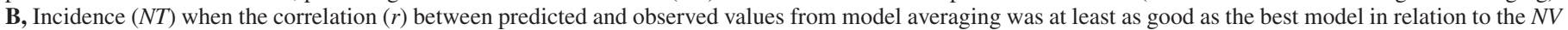
included in models. 
mechanism (i.e., with a fixed number of explanatory variates truly affecting a response variate), the effects of $N V$ in the models to be combined and its interaction with the $N M$ to combined can be explained by this diminished return for additional variates.

An extreme example of combining models from the same dataset (for either training or testing) is found in Shah et al. (2014), who used boosted regression tree (BRT) models to predict the risk of FHB. Averaging of BRT models, which each contained many variates, led to some measures of higher accuracy than the individual BRT. However, unlike in the situation we are exploring, the individual BRT models were each based on different time windows of environmental variables, so that there was no overlap of variates in the different models. However, their results are consistent with our findings that averaging is effective with different variates in the models. Interestingly, the average predictions of the BRT were not more accurate than the predictions from a larger BRT that contained variates from all the time windows, so that that the combined models would have variates from several of the windows.

Combining models derived from different datasets (case 2) may not result in an additional variate in the combined model, unlike combining models derived from the same dataset (case 1). In case 2, two models derived from different datasets may have the exact same set of variates. In this situation, combining models results, in part, in more stable (less extreme) estimates of the $\theta_{l}$ parameters of equation 1 ; averaging is producing intermediate values of the parameters. The likelihood of two models having the exact same variates depends on the relative importance of explanatory variates, correlation among the variates, $N V$ in the model, sample size, and residual errors. Thus, combining models here is not necessarily equivalent to addition of new variates in the model. Rather, the model averaging is attenuating the extreme (over and under) predictions that can be obtained with a single model (Burnham and Anderson 2002). Combining models may not lead to any improvement in prediction accuracy over the best model of those to be combined if the single model has captured the underlying relationship - this is demonstrated by the limited improvement of model averaging over individual models for simulated datasets when $N V>3$. However, for real datasets, we do not know the nature of the underlying relationship, and usually do not have a sufficiently large sample size to develop models with sufficient precision. Therefore, a best model for a given dataset does not necessarily represent the true underlying relationship. Model averaging always resulted in performance being better than the worst-performing single model, though not necessarily better than the best, agreeing with a previous study on assessing performances of watershed models (Boomer et al. 2013). However, the issue is that the best model for one permutated dataset may not be the same one for the others.

In addition to inclusion of additional variates (at least potentially), the relative prediction performance of combining two or three models over the single models for case 2 may also be due to the increased sample size. The importance of sample size for developing and validating models with better predictive power has been emphasized previously (Hastie et al. 2009; Stockwell and Peterson 2002; Vergouwe et al. 2005; Yuan and Yang 2005). There were three sample sizes for developing individual models: 100 in case 1, 67 in case 2 two models, and 50 in case 2 three models. The overall prediction performance (in terms of $r$ ) of individual models increased with increasing sample size, particularly for the dataset with large residual errors (DS2). Yuan and Yang (2005) also showed the importance of the residual variance in the uncertainty of individual selected best models. Thus, improved prediction accuracy from model averaging may be partly due to the improved parameter estimate for a given explaining variate because more data points are used to estimate parameters through the model averaging. Improvement in prediction performance through model averaging over single-model predictions is greatest when combining three models developed from separate samples of size 50, illustrating the value in combining models developed from small datasets to increase their predictive ability. This situation arises when it is impractical to obtain a large number of observations in a single study. Thus, multiple datasets are obtained from different studies but subsequent researchers do not have access to all of the original data, only published models. In this situation, present results demonstrated the value of combining these models because averaging the three best models, each based on a small number of observations (i.e., 50 here), resulted in higher accuracy than using the best model fitted to all 150 points (case 2; data not shown). Thus, it may be worthwhile to develop models from partial datasets several times to assess the importance of variates and to select models for averaging; this approach is essentially the same as developing models via bootstraps (Breiman 1992), a common approach for developing predictive models. Further research is needed to study the relative performance of model averaging and single-model predictions in relation to the sample size and underlying data-generating mechanisms.

This study showed that simple averaging outperformed weighted averaging in providing more accurate predictions (particularly when there were fewer variates in the models to be combined) for all datasets, except for the partial oat data. This is significant because most model averaging involves weights. The simple averaging is sometimes known as the committee method (Hastie et al. 2009) and assumes that each model has equal probability of being the most appropriate one (of the ones being considered). With the small sample sizes typically used in plant protection, the AIC statistic may be estimated imprecisely, negating any advantage of using modelspecific weights. Moreover, the relative importance of individual explanatory variates and, hence, models may vary considerably from sample to sample (dataset to dataset), depending on the other variates in the models and the degree of intercorrelation, especially when there are no dominant explanatory variates influencing the response variable. Thus, model weight based on one sample (where the model is fitted) may not hold for a different (test) sample where the prediction is made. For example, plant disease development in one year and location may be mainly limited by realized temperature and, in another year and location, by realized rainfall, even if the underlying process (not known) is the same for all years and locations. The weight given to a model with temperature and rainfall in the former may not be appropriate for the latter (given that the selected model and estimated parameters in the model are influenced by the range of the variates in each dataset). This variation in the realized strength of relationship of the response variate with explanatory variates may explain the inferior performance of weighted averaging relative to the arithmetic averaging for these scenarios with relatively small sample sizes. The exception in the partial oat data is likely because many important explanatory variates were not included in the dataset $(\mathrm{Xu}$ et al. 2014). Thus, with more models to be combined, there will be variates included that do not improve model performance given the fact that other variates are already included (because the subset regression did not test statistical significance of individual regression parameters). Under this circumstance, weighted averaging will reduce the importance of models with these nonimportant variates. In contrast, simple averaging is not able to do so.

In conclusion, this study demonstrates that combining empirical normal-distribution linear models usually increased predictive power, particularly for cases where there was large residual variation and the number of explanatory variates in the models was small. Combining models derived from the same dataset led to a smaller improvement over single-model predictions than combining models derived from different datasets. It is interesting that a very successful prediction system for potato late blight, BLITECAST, is based on the combination of two independently derived predictors, the Wallin system and the Hyre system. We need to evaluate model averaging using existing models; for instance, apple scab (MacHardy 1996), potato late blight (Hardwick 2006), and FHB in wheat (De Wolf et al. 2003; Moschini and Fortugno 1996; Moschini et al. 2001). 


\section{ACKNOWLEDGMENTS}

$\mathrm{X}$. Xu was supported by the special fund for agro-scientific research in the public interest from Agriculture of Ministry of China (number 201303016) and partially supported by the 111 project from Education of Ministry of China (number B07049).

\section{LITERATURE CITED}

Akaike, H. 1980. Likelihood and the Bayes procedure. Pages 143-166 in: Bayesian Statistics. J. M. Bernardo, M. H. DeGroot, D. V. Lindley, and A. F. M. Smith, eds. University Press, Valencia, Spain.

Boomer, K. M. B., Weller, D. E., Jordan, T. E., Linker, L., Liu, Z.-J., Reilly, J., and Voinov, A. A. 2013. Using multiple watershed models to predict water, nitrogen, and phosphorus discharges to the Patuxent Estuary. J. Am. Water Resour. Assoc. 49:15-39.

Breiman, L. 1992. The little bootstrap and other methods for dimensionality selection in regression: X-fixed prediction error. J. Am. Stat. Assoc. 87: 738-754.

Burnham, K. P., and Anderson, D. R. 2002. Model Selection and Multimodel Inference: A Practical Information-Theoretic Approach. Springer-Verlag, Berlin.

De Wolf, E. D., and Isard, S. A. 2007. Disease cycle approach to plant disease prediction. Annu. Rev. Phytopathol. 45:203-220.

De Wolf, E. D., Madden, L. V., and Lipps, P. E. 2003. Risk assessment models for wheat Fusarium head blight epidemics based on within-season weather data. Phytopathology 93:428-435.

Exbrayat, J. F., Viney, N. R., Frede, H. G., and Breuer, L. 2013. Using multimodel averaging to improve the reliability of catchment scale nitrogen predictions. Geosci. Model Dev. 6:117-125.

Felsenstein, J. 2003. Inferring Phylogenies. Sinauer Associates, Sunderland, MA.

Hansen, B. E. 2007. Least square model averaging. Econometrica 75: 1175-1189.

Hardwick, N. V. 2006. Disease forecasting. Pages 239-267 in: The Epidemiology of Plant Diseases. B. Cooke, D. G. Jones, and B. Kaye, eds. Springer, Dordrecht, The Netherlands.

Hastie, T., Tibshirani, R., and Friedman, J. 2009. The Elements of Statistical Learning: Data Mining, Inference, and Prediction. Springer, New York.

Hoeting, J. A., Madigan, D., Raftery, A. E., and Volinsky, C. T. 1999. Bayesian model averaging: A tutorial. Stat. Sci. 14:382-417.

Hurvich, C. M., and Tsai, C. L. 1990. The impact of model selection on inference in linear regression. Am. Stat. 44:214-217.

Johnson, C. J., Seip, D. R., and Boyce, M. S. 2004. A quantitative approach to conservation planning: Using resource selection functions to map the distribution of mountain caribou at multiple spatial scales. J. Appl. Ecol. 41:238-251.

Johnson, J. B., and Omland, K. S. 2004. Model selection in ecology and evolution. Trends Ecol. Evol. 19:101-108.

Kadane, J. B., and Lazar, N. A. 2004. Methods and criteria for model selection. J. Am. Stat. Assoc. 99:279-290.

Landschoot, S., Waegeman, W., Audenaert, K., Vandepitte, J., Haesaert, G., and De Baets, B. 2012. Toward a reliable evaluation of forecasting systems for plant diseases: A case study using Fusarium head blight of wheat. Plant Dis. 96:889-896.

MacHardy, W. E. 1996. Apple Scab: Biology, Epidemiology, and Management. American Phytopathological Society, St. Paul, MN.
Madden, L. V., Hughes, G., and van den Bosch, F. 2007. The Study of Plant Disease Epidemics. The American Phytopathological Society, St. Paul, MN.

Meads, C., Ahmed, I., and Riley, R. D. 2012. A systematic review of breast cancer incidence risk prediction models with meta-analysis of their performance. Breast Cancer Res. Treat. 132:365-377.

Montgomery, J. M. 2012. Improving predictions using ensemble Bayesian model averaging. Polit. Anal. 20:271-291.

Moschini, R. C., and Fortugno, C. 1996. Predicting wheat head blight incidence using models based in meteorological factors in Pergamino, Argentina. Eur. J. Plant Pathol. 102:211-218.

Moschini, R. C., Pioli, R., Carmona, M., and Sacchi, O. 2001. Empirical predictions of wheat head blight in the northern Argentinean Pampas region. Crop Sci. 41:1541-1545.

Raftery, A. E., Madigan, D., and Hoeting, J. A. 1997. Bayesian model averaging for regression models. J. Am. Stat. Assoc. 92:179-191.

Ralston, A., and Rabinowitz, P. 1978. A First Course in Numerical Analysis. McGraw-Hill, Auckland.

Shah, D. A., De Wolf, E. D., Paul, P. A., and Madden, L. V. 2014. Predicting Fusarium head blight epidemics with boosted regression trees. Phytopathology 104:702-714.

Shah, D. A., Molineros, J. E., Paul, P. A., Willyerd, K. T., Madden, L. V., and De Wolf, E. D. 2013. Predicting Fusarium head blight epidemics with weather-driven pre- and post-anthesis logistic regression models. Phytopathology 103:906-919.

Sillett, T. S., and Holmes, R. T. 2002. Variation in survivorship of a migratory songbird throughout its annual cycle. J. Anim. Ecol. 71:296-308.

Stephens, P. A., Buskirk, S. W., Hayward, G. D., and Martinez del Rio, C. 2005. Information theory and hypothesis testing: A call for pluralism. J. Appl. Ecol. 42:4-12.

Steyerberg, E. W., Eijkemans, M. J. C., and Habbema, J. D. F. 1999. Stepwise selection in small data sets: A simulation study of bias in logistic regression analysis. J. Clin. Epidemiol. 52:935-942.

Stockwell, D. R. B., and Peterson, A. T. 2002. Effects of sample size on accuracy of species distribution models. Ecol. Modell. 148:1-13.

Vergouwe, Y., Steyerberg, E. W., Eijkemans, M. J. C., and Habbema, J. D. F. 2005. Substantial effective sample sizes were required for external validation studies of predictive logistic regression models. J. Clin. Epidemiol. 58: 475-83.

Whittingham, M., Stephens, P., Bradbury, R., and Freckleton, R. 2006. Why do we still use stepwise modelling in ecology and behaviour? J. Anim. Ecol. 75:1182-1189.

Wintle, B. A., McCarthy, M. A., Volinsky, C. T., and Kavanagh, R. P. 2003. The use of Bayesian model averaging to better represent uncertainty in ecological models. Conserv. Biol. 17:1579-1590.

Xu, X.-M., Madden, L. V., and Edwards, S. G. 2014. Modeling the effects of environmental conditions on HT2 and T2 toxin accumulation in field oat grains. Phytopathology 104:57-66.

Xu, X.-M., Madden, L. V., Edwards, S. G., Doohan, F. M., Moretti, A., Hornok, L., and Ritieni, A. 2013. Developing logistic models to relate the accumulation of DON associated with Fusarium head blight to climatic conditions in Europe. Eur. J. Plant Pathol. 137:689-706.

Yang, Y. 2003. Regression with multiple candidate models: Selecting or mixing? Stat. Sin. 13:783-809.

Yuan, Z., and Yang, Y. 2005. Combining linear regression models: When and how? J. Am. Stat. Assoc. 100:1202-1214. 